\title{
On Ideal Convergence of Double Sequences in Probabilistic Normed Spaces
}

\author{
Vijay KUMAR \\ Department of Mathematics, Haryana College of Technology and Management, \\ Kaithal-136027, Haryana, India \\ E-mail:vjy_kaushik@yahoo.com
}

\section{Bernardo LAFUERZA-GUILLÉN}

Departamento de Estadística y Matemática Aplicada, Universidad de Almería, Almería 04120, Spain E-mail: blafuerz@ual.es

\begin{abstract}
The notion of ideal convergence is a generalization of statistical convergence which has been intensively investigated in last few years. For an admissible ideal $\oint \subset \mathbb{N} \times \mathbb{N}$, the aim of the present paper is to introduce the concepts of $\oint$-convergence and $\oint^{\star}$-convergence for double sequences on probabilistic normed spaces (PN spaces for short). We give some relations related to these notions and find condition on the ideal $\oint$ for which both the notions coincide. We also define $\oint$-Cauchy and $\oint^{\star}$ Cauchy double sequences on PN spaces and show that $\oint$-convergent double sequences are $\oint$-Cauchy on these spaces. We establish example which shows that our method of convergence for double sequences on PN spaces is more general.
\end{abstract}

Keywords Ideal convergence, double sequence, statistical convergence, continuous $t$-norm and probabilistic normed spaces

MR(2000) Subject Classification 54E70, 46S70

\section{Introduction}

Fast [1] presented a generalization of the usual concept of sequential limit which he called statistical convergence. Schoenberg [2] and S̆alát [3] gave some basic properties of statistical convergence. Fridy [4] introduced the concept of statistically Cauchy sequence and proved that it is equivalent to statistical convergence. Such an extension has been widely studied from various aspects and also applied to different problems arising in the convergence theory. Mursaleen et al. [5] and Móricz [6] independently extended these concepts from single sequences to double sequences with the help of two dimensional analogue of natural density of subsets of $\mathbb{N} \times \mathbb{N}$. Kostyrko et al. [7] presented an interesting generalization of statistical convergence with the help of an admissible ideal $\oint$ and called it $\oint$-convergence. The idea was extended to double sequences by B. Tripathy and B. C. Tripathy [8] where some properties related to solidity, symmetricity, completeness and denseness are obtained. Kumar [9] defined a closely related 
concept $\oint^{\star}$-convergence for double sequences and proved some results related to $\oint$ and $\oint^{\star}$ convergence. On the other side, Menger [10] proposed the probabilistic concept of the distance by replacing the number $d(p, q)$ as the distance between points $p, q$ by a probability distribution function $F_{p, q}(x)$. He interpreted $F_{p, q}(x)$ as the probability that the distance between $p$ and $q$ is less than $x$. This led to the development of the area now called probabilistic metric spaces. This is S̆herstnev [11] who first used this idea of Menger to introduce the concept of a PN space. In 1993, Alsina et al. [12] presented a new definition of probabilistic normed space which includes the definition of S̆herstnev as a special case. For an extensive view on this subject, we refer [13-19]. Quite recently, Karakus et al. [20] defined statistical analogues of convergence and Cauchy double sequences on PN spaces and gave a useful characterization. Subsequently, V. Kumar and K. Kumar [21] studied $\oint$-Cauchy and $\oint^{\star}$-Cauchy sequences on the same spaces. In this paper, we extend the concepts of $\oint$-convergence, $\oint^{\star}$-convergence and $\oint$-Cauchy from single sequences to double sequences on PN spaces and establish some results related to these notions.

\section{Preliminaries}

Throughout this paper, $\mathbb{N}, \mathbb{R}$ respectively denote the sets of positive integers and real numbers whereas $\mathbb{N} \times \mathbb{N}$ denotes the usual product set. For any set $S, P(S)$ stands for the power set of $S$ and $A^{C}$ denotes the complement of the set $A$.

Definition 2.1 A distribution function (briefly a d.f.) $F$ is a function from the extended reals $\overline{\mathbb{R}}$ into $[0,1]$ such that

(a) it is non-decreasing;

(b) it is left-continuous on $\mathbb{R}$;

(c) $F(-\infty)=0$ and $F(\infty)=1$.

The set of all d.f.'s will be denoted by $\Delta$. The subset of $\Delta$ consisting of proper d.f.'s, namely of those elements $F$ such that $\ell^{+} F(-\infty)=F(-\infty)=0$ and $\ell^{-} F(+\infty)=F(+\infty)=1$ will be denoted by $\mathcal{D}$. A distance distribution function (briefly, d.d.f.) is a d.f. $F$ such that $F(0)=0$. The set of all d.d.f.'s will be denoted by $\Delta^{+}$, while $\mathcal{D}^{+}:=\mathcal{D} \cap \Delta^{+}$will denote the set of proper d.d.f.'s.

Definition 2.2 A triangular norm or, briefly, a $t$-norm is a binary operation $T:[0,1]^{2} \rightarrow$ $[0,1]$ that satisfies the following conditions (see [22]):

(T1) $T$ is commutative, i.e., $T(s, t)=T(t, s)$ for all $s$ and $t$ in $[0,1]$;

(T2) $T$ is associative, i.e., $T(T(s, t), u)=T(s, T(t, u))$ for all $s, t$ and $u$ in $[0,1]$;

(T3) $T$ is nondecreasing, i.e., $T(s, t) \leq T\left(s^{\prime}, t\right)$ for all $t \in[0,1]$ whenever $s \leq s^{\prime}$;

(T4) $T$ satisfies the boundary condition $T(1, t)=t$ for every $t \in[0,1]$.

$T^{*}$ is a continuous $t$-conorm, namely, a continuous binary operation on $[0,1]$ that is related to a continuous $t$-norm through $T^{*}(s, t)=1-T(1-s, 1-t)$. Notice that by virtue of its commutativity, any $t$-norm $T$ is nondecreasing in each place. Some examples of $t$-norms $T$ and its $t$-conorms $T^{*}$ are: $M(x, y)=\min \{x, y\}, \Pi(x, y)=x \cdot y$ and $M^{*}(x, y)=\max \{x, y\}$, $\Pi^{*}(x, y)=x+y-x \cdot y$. 
Using the definitions just given above S̆herstnev [11] defined a PN space as follows:

Definition 2.3 A triplet $(X, \nu, T)$ is called a probabilistic normed space if $X$ is a real vector space, $\nu$ is a mapping from $X$ into $\mathcal{D}$ and for $x \in X$, the d.f. $\nu(x)$ is denoted by $\nu_{x}, \nu_{x}(t)$ is the value of $\nu_{x}$ at $t \in \mathbb{R}$ and $T$ is a $t$-norm. $\nu$ satisfies the following conditions:

(i) $\nu_{x}(0)=0$;

(ii) $\nu_{x}(t)=1$ for all $t>0$ if and only if $x=0$;

(iii) $\nu_{\alpha x}(t)=\nu_{x}\left(\frac{t}{|\alpha|}\right)$ for all $\alpha \in \mathbb{R} \backslash\{0\}$;

(iv) $\nu_{x+y}(s+t) \geq T\left(\nu_{x}(s), \nu_{y}(t)\right)$ for all $x, y \in X$ and $s, t \in \mathbb{R}_{0}^{+}=\{x \in \mathbb{R}: x \geq 0\}$.

Let $(X,\|\cdot\|)$ be a normed space and $\mu \in \mathcal{D}$ with $\mu(0)=0$ and $\mu \neq \epsilon_{0}$, where

$$
\epsilon_{0}(t)= \begin{cases}0, & \text { if } t \leq 0 \\ 1, & \text { if } t>0\end{cases}
$$

For $x \in X, t \in \mathbb{R}$, if we define

$$
\nu_{x}(t)=\mu\left(\frac{t}{\|x\|}\right), \quad x \neq 0,
$$

then in [17], it is proved that $(X, \nu, T)$ is a PN space in the sense of Definition 2.3.

Alsina et al. [12] gave new definition of a PN-Space. Before giving this, we recall for the reader's convenience the concept of a triangle function, that of a $\mathrm{PN}$ space from the point of view of the new definition.

Definition 2.4 A triangle function is a mapping $\tau$ from $\Delta^{+} \times \Delta^{+}$into $\Delta^{+}$such that, for all $F, G, H, K$ in $\Delta^{+}$,

(1) $\tau\left(F, \epsilon_{0}\right)=F$

(2) $\tau(F, G)=\tau(G, F)$;

(3) $\tau(F, G) \leq \tau(H, K)$ whenever $F \leq H, G \leq K$;

(4) $\tau(\tau(F, G), H)=\tau(F, \tau(G, H))$.

Particular and relevant triangle functions are the functions $\tau_{T}, \tau_{T^{*}}$ and those of the form $\Pi_{T}$ which, for any continuous $t$-norm $T$, and any $x>0$, are given by

$$
\begin{aligned}
& \tau_{T}(F, G)(x)=\sup \{T(F(u), G(v)) \mid u+v=x\}, \\
& \tau_{T^{*}}(F, G)(x)=\inf \left\{T^{*}(F(u), G(v)) \mid u+v=x\right\}
\end{aligned}
$$

and

$$
\Pi_{T}(F, G)(x)=T(F(x), G(x)) .
$$

Definition 2.5 ([12]) A probabilistic normed space is a quad-ruple $\left(X, \nu, \tau, \tau^{*}\right)$, where $X$ is a real linear space, $\tau$ and $\tau^{*}$ are continuous triangle functions such that $\tau \leq \tau^{*}$ and the mapping $\nu: X \rightarrow \Delta^{+}$, called the probabilistic norm, satisfies for all $p$ and $q$ in $X$, the conditions

(PN1) $\nu_{p}=\epsilon_{0}$ if and only if, $p=\theta(\theta$ is the null vector in $X)$;

(PN2) $\forall p \in X, \nu_{-p}=\nu_{p}$;

(PN3) $\quad \nu_{p+q} \geq \tau\left(\nu_{p}, \nu_{q}\right)$

(PN4) $\forall \alpha \in[0,1], \nu_{p} \leq \tau^{*}\left(\nu_{\alpha p}, \nu_{(1-\alpha) p}\right)$. 
If a $\mathrm{PN}$ space $\left(X, \nu, \tau, \tau^{*}\right)$ satisfies the following condition

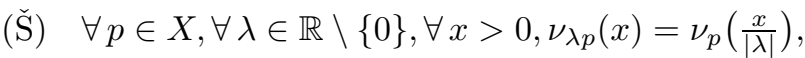

then it is called a S̆herstnev PN space; the condition ( $\breve{\mathrm{S}})$ implies that the best-possible selection for $\tau^{*}$ is $\tau^{*}=\tau_{M}$, which satisfies a stricter version of (PN4), namely,

$$
\forall \lambda \in[0,1], \quad \nu_{p}=\tau_{M}\left(\nu_{\lambda p}, \nu_{(1-\lambda) p}\right) .
$$

It is possible to include the convergence of double sequences in these $\breve{S h}$ erstnev PN spaces. See $[23,24]$ for a recent study of triangle functions.

Definition 2.6 A Menger $\mathrm{PN}$ space under $T$ is a $\mathrm{PN}$ space $\left(X, \nu, \tau, \tau^{*}\right)$ denoted by $(X, \nu, T)$, in which $\tau=\tau_{T}$ and $\tau^{*}=\tau_{T^{*}}$, for some continuous $t$-norm $T$ and its $t$-conorm $T^{*}$.

Theorem $2.7([17]) \quad$ The simple space generated by $(X,\|\cdot\|)$ and by $\mu$ is a Menger PN space under $M$ and also a S̆herstnev PN space. Here $M(x, y):=\min \{x, y\}$.

For further study, by a PN space we mean a PN space in the sense of Definition 2.3. We now give a quick look on the characterization of convergence and Cauchy double sequences on these spaces.

Let $(X, \nu, T)$ be a PN space and $x=\left(x_{i j}\right)$ be a double sequence in $X$. We say that $\left(x_{i j}\right)$ is convergent to $\xi \in X$ with respect to the probabilistic norm $\nu$ if for each $\epsilon>0$ and $\lambda \in(0,1)$ there exists a positive integer $m$ such that $\nu_{x_{i j}-\xi}(\epsilon)>1-\lambda$ whenever $i, j \geq m$. The element $\xi$ is called the ordinary double limit of the sequence $\left(x_{i j}\right)$ and we shall write $\nu$-lim $x_{i j}=\xi$ or $x_{i j} \rightarrow \xi$ as $i, j \rightarrow \infty$ with respect to the probabilistic norm $\nu$.

A double sequence $\left(x_{i j}\right)$ in $X$ is said to be Cauchy with respect to the probabilistic norm $\nu$ if for each $\epsilon>0$ and $\lambda \in(0,1)$ there exist a positive integer $M^{\prime}=M^{\prime}(\epsilon, \lambda)$ and $M=M(\epsilon, \lambda)$ such that $\nu_{x_{i j}-x_{p q}}(\epsilon)>1-\lambda$ whenever $i, p \geq M^{\prime}$ and $j, q \geq M$.

Karakus and Demirci [20] defined the statistical analogues of convergence and Cauchy for double sequences on PN spaces with the help of double natural density given by Mursaleen and Ossama [5] as follows.

Let $K \subset \mathbb{N} \times \mathbb{N}$ and $K(m, n)$ denote the number of $(i, j)$ in $K$ such that $i \leq m$ and $j \leq n$. Then the lower asymptotic density of $K$ is defined by $\underline{\delta}_{2}(K)=\lim \inf _{m, n \rightarrow \infty} \frac{K(m, n)}{m n}$. In case that the sequence $\left(\frac{K(m, n)}{m n}\right)$ has a limit, then we say that $K$ has a double natural density and is defined by $\lim _{m, n \rightarrow \infty} \frac{K(m, n)}{m n}=\delta_{2}(K)$.

Finally, we recall the terminology used in [7] to define $\oint$-convergence. Let $S$ be a non-empty set. A family of sets $\oint \subset P(S)$ is called an ideal in $S$ if and only if (i) $\emptyset \in \oint$; (ii) for each $A, B \in \oint$, we have $A \cup B \in \oint$; (iii) for each $A \in \oint$ and $B \subset A$, we have $B \in \oint$.

A non-empty family of sets $\mathfrak{F} \subset P(S)$ is called a filter on $S$ if and only if (i) $\emptyset \notin \mathfrak{F}$; (ii) for each $A, B \in \mathfrak{F}$, we have $A \cap B \in \mathfrak{F}$; (iii) for each $A \in \mathfrak{F}$ and $B \supset A$, we have $B \in \mathfrak{F}$.

An ideal $\oint$ is called non-trivial if $\oint \neq\{\emptyset\}$ and $S \notin \oint$.

It immediately follows that $\oint \subset P(S)$ is a non-trivial ideal if and only if the class $\Im=\Im(\oint)$ $=\{S-A: A \in \oint\}$ is a filter on $S$. The filter $\Im=\Im(\oint)$ is called the filter associated with the ideal $\oint$.

A non-trivial ideal $\oint \subset P(S)$ is called an admissible ideal in $S$ if and only if it contains all 
singletons, i.e., if it contains $\{\{s\}: s \in S\}$.

An admissible ideal $\oint \subset P(S)$ is said to satisfy the condition (AP) if for every countable family of mutually disjoint sets $\left\{A_{1}, A_{2}, \ldots\right\}$ belonging to $\oint$ there exists a countable family $\left\{B_{1}, B_{2}, \ldots\right\}$ in $\oint$ such that $A_{i} \triangle B_{i}$ is a finite set for each $i \in \mathbb{N}$ and $B=\bigcup_{i=1}^{\infty} B_{i} \in \oint$.

\section{$3 \oint$-convergence of Double Sequences in PN Spaces}

In this section, we define $\oint$-convergence of double sequences on PN spaces and give some of its properties.

Definition 3.1 Let $(X, \nu, T)$ be a $\mathrm{PN}$ space and $\oint \subset P(\mathbb{N} \times \mathbb{N})$ be a non-trivial ideal. A double sequence $x=\left(x_{i j}\right)$ of elements in $X$ is said to be $\oint_{\nu}$-convergent to $\xi \in X$ with respect to the probabilistic norm $\nu$ if for each $\epsilon>0$ and $\lambda \in(0,1)$, the $\operatorname{set}\left\{(i, j) \in \mathbb{N} \times \mathbb{N}: \nu_{x_{i j}-\xi}(\epsilon) \leq\right.$ $1-\lambda\} \in \oint$. The element $\xi$ is called the double $\oint_{\nu}$-limit of the sequence $x=\left(x_{i j}\right)$ and we write $\oint_{\nu}-\lim _{i, j} x_{i j}=\xi$.

Example 3.2 (i) If we take $\oint=\{E \subset \mathbb{N} \times \mathbb{N}: E=(\mathbb{N} \times A) \cup(A \times \mathbb{N})$ for some finite subset $A$ of $\mathbb{N}\}$, then $\oint$-convergence of double sequence on $\mathrm{PN}$ spaces is equivalent to its usual convergence on $\mathrm{PN}$ spaces.

(ii) If we take $\oint=\oint_{\delta_{2}}=\left\{A: A \subset \mathbb{N} \times \mathbb{N}: \delta_{2}(A)=0\right\}$, then $\oint$ is an admissible ideal and the corresponding convergence of double sequences on PN spaces coincides with its statistical convergence on PN spaces.

(iii) Take $\oint=\oint_{\delta^{*}}=\left\{A \subset \mathbb{N} \times \mathbb{N}: \delta^{*}(A)=0\right\}$, where $\delta^{*}(A)$ denotes the logarithmic density of the set $A \subset \mathbb{N} \times \mathbb{N}$ and is defined as follows: A subset $A$ of $\mathbb{N} \times \mathbb{N}$ is said to have logarithmic density $\delta^{*}(A)$ if

$$
\delta^{*}(A)=\lim _{m, n \rightarrow \infty} \frac{1}{s_{m} s_{n}} \sum_{k=1}^{m} \sum_{p=1}^{n} \frac{\chi_{A}(k, p)}{k p},
$$

where $s_{m}=\sum_{k=1}^{m} \frac{1}{k}$ and $s_{n}=\sum_{p=1}^{n} \frac{1}{p}$ exists. One can check $\oint_{\delta^{*}}$ is a non-trivial admissible ideal in $\mathbb{N} \times \mathbb{N}$ and the corresponding convergence coincides with logarithmic statistical convergence of double sequences on $\mathrm{PN}$ spaces.

(iv) The uniform density of a set $A \subset \mathbb{N} \times \mathbb{N}$ is defined as follows. For integers $s, t \geq 0$ and $p, q \geq 1$, let $A(s+1, s+q ; t+1, t+p)=\operatorname{card}\{(m, n) \in A: s+1 \leq m \leq s+q$ and $t+1 \leq n \leq t+p\}$. Put $B_{p, q}=\liminf _{s, t \rightarrow \infty} A(s+1, s+q ; t+1, t+p)$ and $B^{p, q}=\limsup _{s, t \rightarrow \infty} A(s+1, s+q ; t+$ $1, t+p)$. It can be shown that the limits $\underline{u}(A)=\lim _{p, q \rightarrow \infty} \frac{B_{p, q}}{p q}, \bar{u}(A)=\lim _{p, q \rightarrow \infty} \frac{B^{p, q}}{p q}$ exist (see [8]). If $u(A)=\underline{u}(A)=\bar{u}(A)$, then $u(A)$ is called the uniform density of the set $A$. If we take $\oint=\oint_{u}=\{A \subset \mathbb{N} \times \mathbb{N}: u(A)=0\}$, then $\oint_{u}$ is a non-trivial admissible ideal and the corresponding convergence of double sequences coincides with the uniform statistical convergence on PN spaces.

The fact that for each $\epsilon>0$ and $\lambda \in(0,1)$, the set

$$
\left\{(i, j) \in \mathbb{N} \times \mathbb{N}: \nu_{x_{i j}-\xi}(\epsilon) \geq 1+\lambda\right\}=\emptyset,
$$

together with Definition 3.1, implies the following proposition. 
Proposition 3.3 Let $(X, \nu, T)$ be a PN space. For each $\epsilon>0$ and $\lambda \in(0,1)$, the following statements are equivalent:

(i) $\oint_{\nu}-\lim _{i, j} x_{i j}=\xi$;

(ii) $\left\{(i, j) \in \mathbb{N} \times \mathbb{N}: \nu_{x_{i j}-\xi}(\epsilon) \leq 1-\lambda\right\} \in \oint$;

(iii) $\left\{(i, j) \in \mathbb{N} \times \mathbb{N}: \nu_{x_{i j}-\xi}(\epsilon)>1-\lambda\right\} \in \Im(\oint)$;

(iv) $\oint-\lim \nu_{x_{i j}-\xi}(\epsilon)=1$.

The next theorem shows that $\oint_{\nu}$-limit of a double sequence in a $\mathrm{PN}$ space is unique provided it exists.

Theorem 3.4 Let $(X, \nu, T)$ be a PN space and let $x=\left(x_{i j}\right)$ be a double sequence in $X$. If $\left(x_{i j}\right)$ is $\oint$-convergent with respect to the probabilistic norm $\nu$, then its $\oint_{\nu}$-limit is unique.

Proof Suppose that there exist $\xi$ and $\eta$ in $X$ with $\xi \neq \eta, \oint_{\nu}-\lim x_{i j}=\xi$ and $\oint_{\nu}-\lim x_{i j}=\eta$. Let $\lambda>0$. Choose $\gamma \in(0,1)$ such that

$$
T(1-\gamma, 1-\gamma)>1-\lambda
$$

Let $\epsilon>0$ be given. Define

$$
K_{1}=\left\{(i, j) \in \mathbb{N} \times \mathbb{N}: \nu_{x_{i j}-\xi}\left(\frac{\epsilon}{2}\right) \leq 1-\gamma\right\}
$$

and

$$
K_{2}=\left\{(i, j) \in \mathbb{N} \times \mathbb{N}: \nu_{x_{i j}-\eta}\left(\frac{\epsilon}{2}\right) \leq 1-\gamma\right\} .
$$

Since $\oint_{\nu}$-lim $x_{i j}=\xi$ and $\oint_{\nu}$-lim $x_{i j}=\eta$, therefore by definition of a filter we have $K_{1}^{C} \cap K_{2}^{C} \neq \emptyset$. Let $(m, n) \in K_{1}^{C} \cap K_{2}^{C}$. It follows together with (3.1) that

$$
\nu_{\xi-\eta}(\epsilon) \geq T\left(\nu_{x_{m n}-\xi}\left(\frac{\epsilon}{2}\right), \nu_{x_{m n}-\eta}\left(\frac{\epsilon}{2}\right)\right)>T(1-\gamma, 1-\gamma)>1-\lambda .
$$

As $\lambda>0$ was chosen arbitrarily, it follows that $\nu_{\xi-\eta}(\epsilon)=1$ for each $\epsilon>0$. By definition of a PN space we have $\xi-\eta=0$, i.e., $\xi=\eta$. Hence $\oint_{\nu}$-limit of $\left(x_{i j}\right)$ is unique.

Theorem 3.5 Let $(X, \nu, T)$ be a PN space and $x=\left(x_{i j}\right), y=\left(y_{i j}\right)$ be two sequences in $X$.

(i) If $\oint$ contains all sets of the form $\mathbb{N} \times\{n\},\{n\} \times \mathbb{N}$ for $n \in \mathbb{N}$, then $\nu$-lim $x_{i j}=\xi$ implies $\oint_{\nu}-\lim x_{i j}=\xi$;

(ii) If $\oint_{\nu}-\lim x_{i j}=\xi$ and $\oint_{\nu}-\lim y_{i j}=\eta$, then $\oint_{\nu}-\lim \left(x_{i j}+y_{i j}\right)=(\xi+\eta)$;

(iii) If $\oint_{\nu}-\lim x_{i j}=\xi$ and $\alpha$ be a non-zero real number, then $\oint_{\nu}-\lim \alpha x_{i j}=\alpha \xi$. If $\alpha=0$, then the result is true only if the ideal $\oint$ contains all sets of the form $\mathbb{N} \times\{n\},\{n\} \times \mathbb{N}$ for $n \in \mathbb{N}$;

(iv) If $\oint_{\nu}-\lim x_{i j}=\xi$ and $\oint_{\nu}-\lim y_{i j}=\eta$, then $\oint_{\nu}-\lim \left(x_{i j}-y_{i j}\right)=(\xi-\eta)$.

Proof (i) Since $\nu$-lim $x_{i j}=\xi$ so for each $\epsilon>0$ and $\lambda \in(0,1)$, a positive integer $m$ can be found with $\nu_{x_{i j}-\xi}(\epsilon)>1-\lambda$ for every $i, j \geq m$. It follows that the set $A=\{(i, j) \in \mathbb{N} \times \mathbb{N}$ : $\left.\nu_{x_{i j}-\xi}(\epsilon) \leq 1-\lambda\right\} \subset(\mathbb{N} \times\{1,2,3, \ldots, m-1\}) \cup(\{1,2,3, \ldots, m-1\} \times \mathbb{N})$. As the ideal $\oint$ contains all sets of the form $\mathbb{N} \times\{n\},\{n\} \times \mathbb{N}$ for $n \in \mathbb{N}$, so the set on right side and hence $A$ belongs to $\oint$. This shows that $\oint_{\nu}-\lim _{i, j \rightarrow \infty} x_{i j}=\xi$. 
(ii) Take $\epsilon>0$ and $\lambda \in(0,1)$ as arbitrary. Choose $\gamma \in(0,1)$ with the property as in (3.1). If we define the sets $K_{1}=\left\{(i, j) \in \mathbb{N} \times \mathbb{N}: \nu_{x_{i j}-\xi}\left(\frac{\epsilon}{2}\right) \leq 1-\gamma\right\}$ and $K_{2}=\left\{(i, j) \in \mathbb{N} \times \mathbb{N}: F_{x_{i j}-\eta}\left(\frac{\epsilon}{2}\right) \leq\right.$ $1-\gamma\}$, then as in Theorem 3.4, one can see by hypothesis of (ii) that $K_{1}^{C} \cap K_{2}^{C} \in \Im(\oint)$. We next claim that $K_{1}^{C} \cap K_{2}^{C} \subset\left\{(i, j) \in \mathbb{N} \times \mathbb{N}: \nu_{\left(x_{i j}-\xi\right)+\left(y_{i j}-\eta\right)}(\epsilon)>1-\lambda\right\}$. For this let $(m, n) \in K_{1}^{C} \cap K_{2}^{C}$. Now we have, with use of (3.1),

$$
\begin{aligned}
\nu_{\left(x_{m n}-\xi\right)+\left(y_{m n}-\eta\right)}(\epsilon) & \geq T\left(\nu_{x_{m n}-\xi}\left(\frac{\epsilon}{2}\right), \nu_{x_{m n}-\eta}\left(\frac{\epsilon}{2}\right)\right) \\
& >T(1-\gamma, 1-\gamma)>1-\lambda .
\end{aligned}
$$

Hence $K_{1}^{C} \cap K_{2}^{C} \subset\left\{(i, j) \in \mathbb{N} \times \mathbb{N}: \nu_{\left(x_{i j}-\xi\right)+\left(y_{i j}-\eta\right)}(\epsilon)>1-\lambda\right\}$. As $K_{1}^{C} \cap K_{2}^{C} \in \Im(\oint)$, so $\left\{(i, j) \in \mathbb{N} \times \mathbb{N}: \nu_{\left(x_{i j}-\xi\right)+\left(y_{i j}-\eta\right)}(\epsilon) \leq 1-\lambda\right\} \in \oint$. Hence $\oint_{\nu}-\lim \left(x_{i j}+y_{i j}\right)=\xi+\eta$.

(iii) Case 1 Take $\alpha \neq 0$. Since $\oint_{\nu}-\lim x_{i j}=\xi$, so for each $\epsilon>0$ and $\lambda \in(0,1)$, the set $A(\epsilon)=\left\{(i, j) \in \mathbb{N} \times \mathbb{N}: \nu_{x_{i j}-\xi}(\epsilon)>1-\lambda\right\} \in \Im(\oint)$. If $(m, n) \in A(\epsilon)$, then we have

$$
\begin{aligned}
\nu_{\alpha x_{m n}-\alpha \xi}(\epsilon) & =\nu_{x_{m n}-\xi}\left(\frac{\epsilon}{|\alpha|}\right) \geq T\left(\nu_{x_{m n}-\xi}(\epsilon), \nu_{0}\left(\frac{\epsilon}{|\alpha|}-\epsilon\right)\right) \\
& \geq T\left(\nu_{x_{m n}-\xi}(\epsilon), 1\right) \geq \nu_{x_{m n}-\xi}(\epsilon)>1-\lambda
\end{aligned}
$$

as $(m, n) \in A(\epsilon)$. Hence $A(\epsilon) \subset\left\{(i, j) \in \mathbb{N} \times \mathbb{N}: \nu_{\alpha x_{i j}-\alpha \xi}(\epsilon)>1-\lambda\right\}$ and so $\{(i, j) \in \mathbb{N} \times \mathbb{N}$ : $\left.\nu_{\alpha x_{i j}-\alpha \xi}(\epsilon)>1-\lambda\right\} \in \Im(\oint)$. It follows that $\left\{(i, j) \in \mathbb{N} \times \mathbb{N}: \nu_{\alpha x_{i j}-\alpha \xi}(\epsilon) \leq 1-\lambda\right\} \in \oint$. Hence $\oint_{\nu}-\lim \alpha x_{i j}=\alpha \xi$.

Case 2 If $\alpha=0$. Since for $\epsilon>0$ and $\lambda \in(0,1) . \nu_{0 x_{i j}-0 \xi}(\epsilon)=\nu_{\theta}(\epsilon)=1>1-\lambda$, it follows that $\nu$-lim $x_{i j}=0$. Hence by part (i) of the theorem, $\oint_{\nu}-\lim x_{i j}=0$.

(iv) The proof is similar as that of (ii).

Definition 3.6 Let $(X, \nu, T)$ be a PN space. For $x \in X, t>0$ and $r \in(0,1)$, the $(t, r)$ neighborhood of $x$ is defined by

$$
B(x, r, t)=\left\{y \in X: \nu_{x-y}(t)>1-r\right\} .
$$

Definition 3.7 A double sequence $x=\left(x_{i j}\right)$ in a $\mathrm{PN}$ space $(X, \nu, T)$ is said to be bounded if for every $r \in(0,1)$, there exists $t_{0}>0$ such that

$$
\nu_{x_{i j}}\left(t_{0}\right)>1-r
$$

for $i$ and $j$.

Let $\ell_{\infty}^{\nu_{2}}(X)$ denote the space of all bounded double sequences in the PN space $(X, \nu, T)$. For an admissible ideal $\oint$, if $\oint_{b}^{\nu_{2}}(X)$ denotes the set of all $\oint$-convergent and bounded double sequences in a PN space, then we have the following theorem.

Theorem 3.8 Let $(X, \nu, T)$ be a $\mathrm{PN}$ space. For an admissible ideal $\oint, \oint_{b}^{\nu_{2}}(X)$ is a closed linear subspace of $\ell_{\infty}^{\nu_{2}}(X)$.

Proof By Theorem 3.5, it is obvious that $\oint_{b}^{\nu_{2}}(X)$ is a subspace of $\ell_{\infty}^{\nu_{2}}(X)$. We next show that it is closed. Since $\oint_{b}^{\nu_{2}}(X) \subset \overline{\oint_{b}^{\nu_{2}}(X)}$ always, so to prove the result it is sufficient to prove that $\overline{\oint_{b}^{\nu_{2}}(X)} \subset \oint_{b}^{\nu_{2}}(X)$. Let $y \in \overline{\oint_{b}^{\nu_{2}}(X)}$. As $B(y, r, t) \cap \oint_{b}^{\nu_{2}}(X) \neq \emptyset$, so there is an element 
$x \in B(y, r, t) \cap \oint_{b}^{\nu_{2}}(X)$. Let $t>0$ and $\epsilon \in(0,1)$. Choose $r \in(0,1)$ such that

$$
T(1-r, 1-r)>1-\epsilon .
$$

Since $x \in B(y, r, t) \cap \oint_{b}^{\nu_{2}}(X)$, there is a set $K \subseteq \mathbb{N} \times \mathbb{N}$ with $K \in \Im(\oint)$ such that for all $i, j \in K$,

$$
\nu_{y_{i j}-x_{i j}}\left(\frac{t}{2}\right)>1-r \quad \text { and } \quad \nu_{x_{i j}}\left(\frac{t}{2}\right)>1-r .
$$

For $i, j \in K$, we have by (3.2) and (3.3),

$$
\nu_{y_{i j}}(t)=\nu_{y_{i j}-x_{i j}+x_{i j}}(t) \geq T\left(\nu_{y_{i j}-x_{i j}}\left(\frac{t}{2}\right), \nu_{x_{i j}}\left(\frac{t}{2}\right)\right) \geq T((1-r),(1-r))>1-\epsilon .
$$

It follows that $K \subset\left\{(i, j) \in \mathbb{N} \times \mathbb{N}: \nu_{y_{i j}}(t)>1-\epsilon\right\}$. Since $K \in \Im(\oint)$, by definition, $\left\{(i, j) \in \mathbb{N} \times \mathbb{N}: \nu_{y_{i j}}(t)>1-\epsilon\right\} \in \Im(\oint)$, and therefore $\left\{(i, j) \in \mathbb{N} \times \mathbb{N}: \nu_{y_{i j}}(t) \leq 1-\epsilon\right\} \in \oint$. This shows that $y \in \oint_{b}^{\nu_{2}}(X)$ and hence $\overline{\oint_{b}^{\nu_{2}}(X)} \subset \oint_{b}^{\nu_{2}}(X)$. This completes the proof of the theorem.

\section{$4 \oint^{\star}$-convergence of Double Sequences in PN Spaces}

In this section we define a more generalized convergence which is closely related to $\oint$-convergence. Recently Karakus et al. [20] proved that in a PN space $(X, \nu, T)$, a double sequence $x=\left(x_{i j}\right)$ is statistically convergent to $\xi$ if and only if there exists a subset $K \subset \mathbb{N} \times \mathbb{N}$ such that $\delta_{2}(K)=1$ and $\nu$ - $\lim _{(i, j) \in K, i, j \rightarrow \infty} x_{i j}=\xi$. We use this well-known result of statistical convergence to introduce the notion of $\oint^{\star}$-convergence in PN spaces as follows.

Definition 4.1 A double sequence $x=\left(x_{i j}\right)$ is said to be $\oint_{\nu}^{\star}$-convergent to an element $\xi \in X$ if and only if there exist a set $K \in \Im(\oint)$ such that $\nu$ - $\lim _{(i, j) \in K, i, j \rightarrow \infty} x_{i j}=\xi$.

Theorem 4.2 Let $(X, \nu, T)$ be a PN space and $x=\left(x_{i j}\right)$ be a double sequence in $X$. If the ideal $\oint$ contains all sets of the form $H \times \mathbb{N}, \mathbb{N} \times H$, where $H$ is a finite subset of $\mathbb{N}$, then $\oint_{\nu}^{\star}-\lim x_{i j}=\xi$ implies $\oint_{\nu}-\lim x_{i j}=\xi$.

Proof Since $\oint_{\nu}^{\star}-\lim x_{i j}=\xi$, so for $\epsilon>0$ and $\lambda \in(0,1)$, there are a set $K=\{(i, j) ; i, j=$ $1,2, \ldots\} \in \Im(\oint)$ and a positive integer $n_{1}$ such that

$$
\nu_{x_{i j}-\xi}(\epsilon)>1-\lambda
$$

whenever $i, j \geq n_{1}$ and $(i, j) \in K$. Let $A=\left\{1,2, \ldots, n_{1}-1\right\} ; B=\left\{(i, j) \in K: \nu_{x_{i j}-\xi}(\epsilon) \leq\right.$ $1-\lambda\}$. Then it is clear that $B \subset(A \times \mathbb{N}) \cup(\mathbb{N} \times A)$ and therefore belongs to $\oint$. Also $K \in \Im(\oint)$ therefore $K=\mathbb{N} \times \mathbb{N} \backslash H$ for some $H \in \oint$. Obviously, the set $\left\{(i, j) \in \mathbb{N} \times \mathbb{N}: \nu_{x_{i j}-\xi}(\epsilon) \leq\right.$ $1-\lambda\} \subset B \cup H$ and so the theorem is proved.

Example 4.3 Let $(\mathbb{R},|\cdot|)$ denote the space of real numbers with the usual norms. We define $T(a, b)=a b$ and $\nu_{x}(t)=\frac{t}{t+|x|}$ where $x \in \mathbb{R}$ and $t \geq 0$. Then it is easy to see that $(\mathbb{R},|\cdot|)$ is a $\mathrm{PN}$ space.

Let $\mathbb{N}=\bigcup_{i=1}^{\infty} N_{i}$ be a disjoint decomposition of $\mathbb{N}$ such that each $N_{i}$ is an infinite set. Then it is obvious that $\mathbb{N} \times \mathbb{N}=\bigcup_{i=1}^{\infty} \bigcup_{j=1}^{\infty}\left(N_{i} \times N_{j}\right)$ is a disjoint decomposition of $\mathbb{N} \times \mathbb{N}$. If we take 
for some positive integers $p$ and $q$,

$$
\oint=\left\{A \subset \mathbb{N} \times \mathbb{N}: A \subset\left(\mathbb{N} \times\left(\bigcup_{i=1}^{p} N_{i}\right)\right) \cup\left(\left(\bigcup_{j=1}^{q} N_{j}\right) \times \mathbb{N}\right)\right\}
$$

then it is easy to see that $\oint$ is an admissible ideal in $\mathbb{N} \times \mathbb{N}$ such that $\oint$ contains the sets of the form $H \times \mathbb{N}, \mathbb{N} \times H$ where $H$ is a finite subset of $\mathbb{N}$. We define a sequence $x=\left(x_{m n}\right)$ as follows. For $(m, n) \in N_{i} \times N_{j}$, define $x_{m n}=\frac{1}{i}+\frac{1}{j}$ where $i, j=1,2,3 \ldots$ Now $\nu_{x_{m n}}(t)=\frac{t}{t+\left|x_{m n}\right|} \rightarrow 1$ as $m, n \rightarrow \infty$ and therefore first part of Theorem 3.5 we have $\oint_{\nu}-\lim x_{m n}=1$.

Next we prove that $x=\left(x_{m n}\right)$ is not $\oint_{\nu}^{\star}$-convergent. Suppose it is $\oint_{\nu}^{\star}$-convergent sequence. By definition, there exists a set $K=\{(m, n): m, n=1,2,3, \ldots\} \in \Im(\oint)$ such that $\nu$ - $\lim _{(m, n) \in K, m, n \rightarrow \infty} x_{m n}=\xi$. Since $K \in \Im(\oint)$, therefore there is a set $B \in \oint$ such that $K=\mathbb{N} \times \mathbb{N} \backslash B$. By definition of an ideal there exist positive integers $p$ and $q$ such that

$$
B \subset\left(\mathbb{N} \times\left(\bigcup_{i=1}^{p} N_{i}\right)\right) \cup\left(\left(\bigcup_{j=1}^{q} N_{j}\right) \times \mathbb{N}\right) .
$$

It follows that the set $\left\{N_{p+1} \times N_{q+1}\right\} \subset K$. Since $\left\{N_{p+1} \times N_{q+1}\right\}$ is an infinite set so $K$ contains $\frac{1}{p+1}+\frac{1}{q+1}$ as infinite numbers of terms. This shows that $\oint_{\nu}^{\star}-\lim _{m, n \rightarrow \infty} x_{m n}$ does not exist and therefore we arrive at a contradiction. Hence, the sequence $x=\left(x_{m n}\right)$ is not $\oint_{\nu}^{\star}$-convergent.

Theorem 4.4 Let $(X, \nu, T)$ be a PN space and $x=\left(x_{i j}\right)$ be a double sequence in $X$. If the ideal $\oint$ satisfies the property (AP), then $\oint_{\nu}$-convergence implies $\oint_{\nu}^{\star}$-convergence.

Proof Suppose that the ideal $\oint$ satisfies the condition $(A P)$. Let $x=\left(x_{i j}\right)$ be a double sequence in $X$ such that $\oint_{\nu}-\lim x_{i j}=\xi$ for some $\xi \in X$. Then for each $\epsilon>0$ and $\lambda \in(0,1)$ the set $\left\{(i, j) \in \mathbb{N} \times \mathbb{N}: \nu_{x_{i j}-\xi}(\epsilon) \leq 1-\lambda\right\} \in \oint$. For $n \in \mathbb{N}$, we define the set $A_{n}$ as follows:

$$
A_{n}=\left\{(i, j) \in \mathbb{N} \times \mathbb{N}: 1-\frac{1}{n}<\nu_{x_{i j}-\xi}(\epsilon) \leq 1-\frac{1}{n+1}\right\} .
$$

It is clear that $\left\{A_{1}, A_{2}, \ldots\right\}$ is a countable family of mutually disjoint sets belonging to $\oint$ and therefore by the condition (AP) there is a countable family of sets $\left\{B_{1}, B_{2}, \ldots\right\} \in \oint$ such that $A_{i} \triangle B_{i}$ is a finite set for each $i \in \mathbb{N}$ and $B=\bigcup_{i=1}^{\infty} B_{i} \in \oint$. Since $B \in \oint$ so there is a set $K \in \Im(\oint)$, such that $K=\mathbb{N} \times \mathbb{N} \backslash B$. Now to prove the result it is sufficient to prove that $\nu$ - $\lim _{i, j \in K, i, j \rightarrow \infty} x_{i j}=\xi$.

Let $\epsilon>0$ be given and take $\lambda \in(0,1)$. Choose a positive integer $q$ such that $\frac{1}{q}<\lambda$. Then we have

$$
\left\{(i, j) \in \mathbb{N} \times \mathbb{N}: \nu_{x_{i j}-\xi}(\epsilon) \leq 1-\lambda\right\} \subset\left\{(i, j) \in \mathbb{N} \times \mathbb{N}: \nu_{x_{i j}-\xi}(\epsilon) \leq 1-\frac{1}{q}\right\} \subset \bigcup_{i=1}^{q} A_{i} .
$$

Since $A_{i} \triangle B_{i}$ is a finite set for each $i=1,2, \ldots, q+1$, therefore there exist a positive integer $n_{0}$ such that $\left\{\left\{\bigcup_{i=1}^{q+1} B_{i}\right\} \cap\left\{(i, j) \in \mathbb{N} \times \mathbb{N}: i, j \geq n_{0}\right\}\right\}=\left\{\left\{\bigcup_{i=1}^{q+1} A_{i}\right\} \cap\left\{(i, j) \in \mathbb{N} \times \mathbb{N}: i, j \geq n_{0}\right\}\right\}$. If $i, j>n_{0}$ and $(i, j) \in K$, then $(i, j) \notin B$. This implies that $(i, j) \notin \bigcup_{i=1}^{q} B_{i}$, and therefore $(i, j) \notin \bigcup_{i=1}^{q} A_{i}$. Hence for every $i, j \geq n_{0},(i, j) \in K$, we have, by $(4.1), \nu_{x_{i j}-\xi}(\epsilon)>1-\lambda$. This completes the proof of the theorem. 


\section{$5 \oint$-Cauchy and $\oint^{\star}$-Cauchy Double Sequences in PN Spaces}

Dems [25] defined the $\oint$-Cauchy sequence in a metric space. In this section we introduce $\oint$-Cauchy and $\oint^{\star}$-Cauchy for double sequences on PN spaces as follows.

Definition 5.1 Let $(X, \nu, T)$ be a PN space. A double sequence $x=\left(x_{i j}\right)$ is said to be $\oint_{\nu}$ Cauchy sequence if for each $\epsilon>0$ and $\lambda \in(0,1)$, there exists $(m, n) \in \mathbb{N} \times \mathbb{N}$ such that the set $\left\{(i, j) \in \mathbb{N} \times \mathbb{N}: \nu_{x_{i j}-x_{m n}}(\epsilon) \leq 1-\lambda\right\} \in \oint$.

Definition 5.2 Let $(X, \nu, T)$ be a PN space. A double sequence $x=\left(x_{i j}\right)$ is said to be $\oint_{\nu}^{\star}$ Cauchy if and only if there exist a set $K=\{(i, j)\}, i, j=1,2,3, \ldots\} \in \Im(\oint)$ such that the subsequence $\left(x_{i_{k}, j_{k}}\right)$ is an ordinary Cauchy sequence with respect to the probabilistic norm $\nu$.

Theorem 5.3 Let $(X, \nu, T)$ be a PN space. If a double sequence $x=\left(x_{i j}\right)$ is $\oint_{\nu}$-convergent, then it is $\oint_{\nu}$-Cauchy.

Proof Let $x=\left(x_{i j}\right)$ be $\oint_{\nu}$-convergent to $\xi$. Take $\epsilon>0$ and $\lambda \in(0,1)$ to be arbitrary. Choose $\gamma \in(0,1)$ such that $(3.1)$ holds. Since $\oint_{\nu}$-lim $x_{i j}=\xi$, it follows that for each $\epsilon>0$ and $\lambda \in(0,1)$, the set $A=\left\{(i, j) \in \mathbb{N} \times \mathbb{N}: \nu_{x_{i j}-\xi}(\epsilon) \leq 1-\gamma\right\} \in \oint$ and hence $A^{C}=$ $\left\{(i, j) \in \mathbb{N} \times \mathbb{N}: \nu_{x_{i j}-\xi}(\epsilon)>1-\gamma\right\}$ is a non-empty set in $\Im(\oint)$. Let $(m, n) \in A^{C}$. If we take $B=\left\{(i, j) \in \mathbb{N} \times \mathbb{N}: \nu_{x_{i j}-x_{m n}}(\epsilon) \leq 1-\lambda\right\}$, then to prove the result it is sufficient to prove that $B$ is contained in $A$. If $(k, l) \in B$, then we have $\nu_{x_{k l}-x_{m n}}(\epsilon) \leq 1-\lambda$. Furthermore, we have either $\nu_{x_{k l}-\xi}\left(\frac{\epsilon}{2}\right) \leq 1-\gamma$ or $\nu_{x_{k l}-\xi}\left(\frac{\epsilon}{2}\right)>1-\gamma$. If $\nu_{x_{k l}-\xi}\left(\frac{\epsilon}{2}\right) \leq 1-\gamma$, then $(k, l) \in A$. Assume that $\nu_{x_{k l}-\xi}\left(\frac{\epsilon}{2}\right)>1-\gamma$, then

$$
1-\lambda \geq \nu_{x_{k l}-x_{m n}}(\epsilon) \geq T\left(\nu_{x_{k l}-\xi}\left(\frac{\epsilon}{2}\right), \nu_{x_{m n}-\xi}\left(\frac{\epsilon}{2}\right)\right)>T(1-\gamma, 1-\gamma)>1-\lambda,
$$

which is not possible. Hence, $B \subset A$ and therefore $B \in \oint$. This shows that $\left(x_{i j}\right)$ is $\oint_{\nu}$ Cauchy.

Theorem 5.4 Let $(X, \nu, T)$ be a PN space. If some subsequence of a $\oint_{\nu}$-Cauchy double sequence $x=\left(x_{i j}\right)$ is $\oint_{\nu}$-convergent, then the sequence $x=\left(x_{i j}\right)$ is $\oint_{\nu}$-convergent.

Proof Take $\epsilon>0$ and $\lambda \in(0,1)$ to be arbitrary. Choose $\gamma \in(0,1)$ such that

$$
T(1-\gamma, 1-\gamma)>1-\lambda
$$

Since $\left(x_{i j}\right)$ is a $\oint_{\nu}$-Cauchy sequence so there exists a pair $(m, n)$ in $\mathbb{N} \times \mathbb{N}$ such that the set $A=\left\{(i, j) \in \mathbb{N} \times \mathbb{N}: \nu_{x_{i j}-x_{m n}}\left(\frac{\epsilon}{2}\right)>1-\gamma\right\} \in \Im(\oint)$. Suppose that $\left(x_{i_{k} j_{k}}\right)$ is a subsequence of $\left(x_{i j}\right)$ such that $\oint_{\nu}-\lim x_{i_{k} j_{k}}=\xi$. It follows that the set $B=\left\{\left(i_{k}, j_{k}\right) \in \mathbb{N} \times \mathbb{N}: \nu_{x_{i_{k} j_{k}}-\xi}\left(\frac{\epsilon}{2}\right)>\right.$ $1-\gamma\} \in \Im(\oint)$. Let $C=\left\{(i, j) \in \mathbb{N} \times \mathbb{N}: \nu_{x_{i j}-\xi}(\epsilon)>1-\lambda\right\}$. We claim that $A \cap B \subset C$. If $\left(i_{k}, j_{k}\right) \in A \cap B$, then we have

$$
\nu_{x_{i_{k} j_{k}}-x_{m n}}\left(\frac{\epsilon}{2}\right)>1-\gamma \text { and } \nu_{x_{i_{k} j_{k}}-\xi}\left(\frac{\epsilon}{2}\right)>1-\gamma .
$$

Since

$$
\nu_{x_{i_{k} j_{k}}-\xi}(\epsilon) \geq T\left(\nu_{x_{i_{k} j_{k}}-x_{m n}}\left(\frac{\epsilon}{2}\right), \nu_{m n-\xi}\left(\frac{\epsilon}{2}\right)\right)>T(1-\gamma, 1-\gamma)>1-\lambda,
$$

it follows that $\left(i_{k}, j_{k}\right) \in C$. Hence $A \cap B \subset C$ and therefore the theorem is proved. 
Theorem 5.5 Let $(X, \nu, T)$ be a PN space. If a double sequence $x=\left(x_{i j}\right)$ is $\oint_{\nu}^{\star}$-convergent, then it is $\oint_{\nu}^{\star}$-Cauchy sequence.

\section{Conclusion}

In this paper we study ideal convergence and related notions for double sequences on $\mathrm{PN}$ spaces and observe that giving particular choice to the ideal $\oint$, we obtain the corresponding usual and statistical convergence. Since ideal convergence is a generalization of statistical convergence and the latter is used to find optimal paths so the results presented in this paper can be used as theoretical tools to study optical paths where the norm is probabilistic rather than crisp. Furthermore, every ordinary norm induces a probabilistic norm, the results obtained here are more general than the corresponding of normed spaces. The definitions and results presented in this work remain valid in a more general context, that of general PN spaces in the sense of Definition 2.5.

Acknowledgements We would like to express our gratitude to the referees of the paper for their useful comments and suggestions.

\section{References}

[1] Fast, H.: Surla convergence statistique. Colloq. Math., 2, 241-244 (1951)

[2] Schoenberg, I. J.: The integrability of certain function and related summability methods. Amer. Math. Monthly, 66, 361-375 (1959)

[3] S̆alát, T.: On statistically convergent sequences of real numbers. Math. Slovaca, 30, 139-150 (1980)

[4] Fridy, J. A.: On statistical convergence. Analysis, 5(4), 301-313 (1985)

[5] Mursaleen, M., Osama, H. H. E.: Statistical convergence of double sequences. J. Math. Anal. Appl., 288, 223-231 (2003)

[6] Móricz, F.: Statistical convergence of multiple sequences. Arch. Math., 81, 82-89 (2003)

[7] Kostyrko, P., S̆alát T., Wilczynski, W.: $\oint$-convergence. Real Anal. Exchange, 26, 669-686 (2000/2001)

[8] Tripathy, B., Tripathy, B. C.: On $\oint$-convergence of double sequences. Soochow J. Math., 31, 549-560 (2005)

[9] Kumar, V.: On $\oint \& \oint^{\star}$-convergence of double sequences. Math. Commun., 12, 171-181 (2007)

[10] Menger, K.: Statistical metrics. Proc. Nat. Acad. Sci. USA, 28, 535-537 (1942)

[11] S̆herstnev, A. N.: Random normed spaces: Problems of completeness. Kazan Gos. Univ. Ucen. Zap., 122, 3-20 (1962)

[12] Alsina, C., Schweizer, B., Sklar1, A.: On the definition of a probabilistic normed space. Aequationes Math., 46, 91-98(1993)

[13] Schweizer, B., Sklar1, A.: Statistical metric spaces. Pacific J. Math., 10, 314-344 (1960)

[14] Frank, M. J., Schweizer, B.: On the duality of generalized infimal and supremal convolutions. Rend. Mat., 12(6), 1-23 (1979)

[15] Constantin, G., Istratescu, I.: Elements of Probabilistic Analysis, Kluwer, 1989

[16] Alsina, C., Schweizer, B., Sklar, A.: Continuity properties of probabilistic norms. J. Math. Anal. Appl., 208, 446-452 (1997)

[17] Lafuerza-Guillén, B., Rodríguez-Lallena, J. A., Sempi, C.: Some classes of probabilistic normed spaces. Rend. Mat., 17, 237-252 (1997)

[18] Schweizer, B., Sklar, A.: Probabilistic Metric Spaces, Elsevier, New York, 1983; 2nd ed., Dover Publication, Mineola, NY, 2005

[19] Asadollah, A., Nourouzi, K.: Convex sets in probabilistic normed spaces. Chaos, Solitons and Fractals, 36(2), 322-328 (2008)

[20] Karakus, S., Demirci, K.: Statistical convergence of double sequences on probabilistic normed spaces. Int. J. Math. Math. Sci., Article ID 14737, 11 pages (2007) 
[21] Kumar, V., Kumar, K.: On $\oint$ and $\oint^{\star}$-Cauchy sequences in probabilistic normed spaces. Mathematical Sciences, 2(1), 47-58 (2008)

[22] Klement, E. P., Mesiar, R., Pap, E.: Triangular Norms, Kluwer, Dordrecht, 2000

[23] Saminger-Platz, S., Sempi, C.: A primer on triangle functions I. Aequationes Math., 76, 201-240 (2008)

[24] Saminger-Platz, S., Sempi, C.: A primer on triangle functions II. Aequationes Math., 80(3), 239-268 (2010)

[25] Dems, K.: On $\oint$-Cauchy sequences. Real Anal. Exchange, 30(1), 123-128 (2004/2005) 